\title{
Thermal stability of interstitial and substitutional Mn in ferromagnetic (Ga,Mn)As
}

\author{
T. A. L. Lima $\odot,{ }^{1}$ U. Wahl,,${ }^{1,2}$ A. Costa, ${ }^{2}$ V. Augustyns,,${ }^{1}$ K. W. Edmonds, ${ }^{3}$ B. L. Gallagher, ${ }^{3}$ R. P. Campion, ${ }^{3}$ \\ J. P. Araújo, ${ }^{4}$ J. G. Correia, ${ }^{2}$ M. R. da Silva,${ }^{2}$ K. Temst, ${ }^{1}$ A. Vantomme,,${ }^{1}$ and L. M. C. Pereira ${ }^{1},{ }^{*}$ \\ ${ }^{1}$ KU Leuven, Instituut voor Kern- en Stralingsfysica, 3001 Leuven, Belgium \\ ${ }^{2}$ Centro de Ciências e Tecnologias Nucleares, Instituto Superior Técnico, Universidade de Lisboa, 2686-953 Sacavém, Portugal \\ ${ }^{3}$ School of Physics and Astronomy, University of Nottingham, Nottingham NG7 2RD, United Kingdom \\ ${ }^{4}$ IFIMUP and IN-Institute of Nanoscience and Nanotechnology, Universidade do Porto, 4169-007 Porto, Portugal
}

(Received 8 July 2019; revised manuscript received 4 September 2019; published 7 October 2019)

\begin{abstract}
In (Ga,Mn)As, a model dilute magnetic semiconductor, the electric and magnetic properties are strongly influenced by the lattice sites occupied by the Mn atoms. In particular, the highest Curie temperatures are achieved upon thermal annealing in a narrow temperature window around $200{ }^{\circ} \mathrm{C}$, by promoting the diffusion of interstitial Mn towards the surface. In this work, we determined the thermal stability of both interstitial and substitutional $\mathrm{Mn}$ in ferromagnetic $(\mathrm{Ga}, \mathrm{Mn}) \mathrm{As}$ thin films, using the emission channeling technique. At a higher Mn concentration, the temperatures at which substitutional and interstitial Mn become mobile not only decrease, but also become closer to each other. These findings advance our understanding of selfcompensation in $(\mathrm{Ga}, \mathrm{Mn}) \mathrm{As}$ by showing that the strong dependence of the Curie temperature on annealing temperature around $200{ }^{\circ} \mathrm{C}$ is a consequence of balance between diffusion of interstitial $\mathrm{Mn}$ and segregation of substitutional Mn.
\end{abstract}

DOI: $10.1103 /$ PhysRevB.100.144409

\section{INTRODUCTION}

(Ga,Mn)As, a dilute magnetic semiconductor (DMS), is a model system for studying carrier-mediated ferromagnetism in semiconductors and the associated spintronic phenomena [1-3]. In ferromagnetic (Ga,Mn)As thin films, which are typically grown by low-temperature molecular-beam epitaxy (LT-MBE), Mn atoms mainly occupy Ga sites with a minority fraction occupying interstitial sites [4-6]. Substitutional Mn atoms $\left(\mathrm{Mn}_{\mathrm{s}}\right)$ provide both the localized magnetic moment and the itinerant holes that mediate the magnetic coupling. On the other hand, interstitial $\mathrm{Mn}$ atoms $\left(\mathrm{Mn}_{\mathrm{i}}\right)$ have a doubly compensating effect: magnetic compensation since $\mathrm{Mn}_{\mathrm{i}}$ couple antiferromagnetically with $\mathrm{Mn}_{\mathrm{s}}$, and electric compensation since $\mathrm{Mn}_{\mathrm{i}}$ are double donors [5]. At a given Mn concentration, the substitutional-to-interstitial ratio strongly influences the hole concentration, the Fermi level, and the effective magnetization provided by noncompensated $\mathrm{Mn}_{\mathrm{s}}$ moments [1-7].

Previous studies on (Ga,Mn)As have focused on a careful optimization of the synthesis and postgrowth treatments, aiming to improve two key properties: the Curie temperature $\left(T_{\mathrm{C}}\right)$ and the magnetization [4-8]. In these studies at high $\mathrm{Mn}$ concentration (several percent), interstitial $\mathrm{Mn}$ was found to out-diffuse during thermal annealing in the $160-200{ }^{\circ} \mathrm{C}$ temperature range [7-10]. The highest $T_{\mathrm{C}}$ in (Ga,Mn)As $(188 \mathrm{~K})$ has been achieved for a $\mathrm{Mn}$ concentration of $\sim 12 \%$ after growth at low temperature $\left(T_{G} \sim 200^{\circ} \mathrm{C}\right)$, followed by thermal annealing in air at $160^{\circ} \mathrm{C}[7,8]$. Understanding the diffusion of interstitial Mn (i.e., its thermal stability) is crucial, as it defines the lower limit for the optimum annealing temperature

*lino.pereira@kuleuven.be window. In particular, the binding energy of the interstitial $\mathrm{Mn}$ in complexes such as $\mathrm{Mn}_{\mathrm{s}}-\mathrm{Mn}_{\mathrm{i}}$ pairs and $\mathrm{Mn}_{\mathrm{s}}-\mathrm{Mn}_{\mathrm{i}}-\mathrm{Mn}_{\mathrm{s}}$ triplets [11,12], which can be as high as $0.8 \mathrm{eV}[10,13]$, contributes to its thermal stability. Therefore, since the fraction of $\mathrm{Mn}$ atoms in $\mathrm{Mn}_{\mathrm{s}}-\mathrm{Mn}_{\mathrm{i}}$ pairs is expected to increase with increasing Mn concentration, so would the thermal stability of interstitial Mn. However this dependence has not been studied so far. On the other hand, the diffusion and segregation of substitutional $\mathrm{Mn}$ also plays a central role on the magnetic properties of $(\mathrm{Ga}, \mathrm{Mn}) \mathrm{As}$, as it defines the upper limit for optimum annealing. Experiments based on ion channeling showed that part of the substitutional Mn is converted to a nonsubstitutional (random) component at annealing temperatures as low as $\sim 280^{\circ} \mathrm{C}$ [6]. Additionally, a detailed study of the dependence of $T_{\mathrm{C}}$ on annealing temperature revealed a decrease in $T_{\mathrm{C}}$ with increasing annealing temperature in the range $160-220^{\circ} \mathrm{C}$, suggesting an onset of segregation of $\mathrm{Mn}_{\mathrm{s}}$ [9]. However, these temperatures are well below the wellunderstood regime of secondary phase formation $\left(>400{ }^{\circ} \mathrm{C}\right)$ $[11,12,14,15]$. Moreover, direct evidence for $\mathrm{Mn}_{\mathrm{s}}$ segregation around $200^{\circ} \mathrm{C}$ is still lacking.

The diffusion of interstitial $\mathrm{Mn}$ and the segregation of substitutional Mn in (Ga,Mn)As remain poorly understood. In this paper, we address this gap in understanding by studying the thermal stability of $\mathrm{Mn}$ in ferromagnetic (Ga,Mn)As with a concentration of $1 \%$ and $5 \%$. Using the emission channeling technique, we observe that, with increasing Mn concentration, the temperatures at which $\mathrm{Mn}_{\mathrm{s}}$ and $\mathrm{Mn}_{\mathrm{i}}$ become mobile not only decrease, but also become closer to each other. These results show that the narrow annealing temperature window in which the $T_{\mathrm{C}}$ and magnetization can be optimized in ( $\mathrm{Ga}, \mathrm{Mn}$ )As results from a fine balance between diffusion of $\mathrm{Mn}_{\mathrm{i}}$ and segregation of $\mathrm{Mn}_{\mathrm{s}}$. 
TABLE I. Sample and implantation details. All implantations were performed at room temperature. The peak concentrations $\left(x_{p}\right)$, projected ion range $\left(R_{p}\right)$, and straggling $(\sigma)$ were estimated using SRIM-2008 code [22]. The sample thickness, implantation energy, and angle are denoted, respectively, by $t, E$, and $\theta$.

\begin{tabular}{|c|c|c|c|c|c|c|c|c|c|}
\hline Sample (Mn) & $t(\mathrm{~nm})$ & Isotope & $E(\mathrm{keV})$ & $R_{p}(\mathrm{~nm})$ & $\sigma(\mathrm{nm})$ & $x_{p}\left(\mathrm{~cm}^{-3}\right)$ & Fluence $\left(\mathrm{cm}^{-2}\right)$ & $\theta(\operatorname{deg})$ & \\
\hline B & $5 \%$ & 200 & ${ }^{56} \mathrm{Mn}$ & 30 & 21 & 11 & $2.4 \times 10^{18}$ & $7 \times 10^{12}$ & 17 \\
\hline
\end{tabular}

\section{EXPERIMENTAL DETAILS}

The emission channeling technique makes use of charged particles emitted by a decaying radioactive isotope, usually $\beta^{-}$decay electrons or conversion electrons [16]. These particles are emitted isotropically during decay and are channeled along the screened Coulomb potential of atomic rows and planes. Along low-index crystal directions of single crystals or epilayers, this anisotropic scattering results in welldefined channeling or blocking effects. Because these effects strongly depend on the initial position of the emitted particles, they produce emission patterns which are characteristic of the lattice sites occupied by the probe atoms. Emission channeling experiments were performed by implanting a low concentration $(<0.05 \%)$ of radioactive ${ }^{56} \mathrm{Mn}$ (half-life $t_{1 / 2}=2.56 \mathrm{~h}$ ) or ${ }^{73} \mathrm{As}$ (half-life $t_{1 / 2}=80 \mathrm{~d}$ ) into $(\mathrm{Ga}, \mathrm{Mn}) \mathrm{As}$ thin films. Angular-dependent emission patterns are recorded along various crystallographic axes using a position- and energy-sensitive detection system similar to that described in Ref. [17]. The theoretical emission patterns for probes occupying a large variety of possible lattice sites are calculated using the many-beam formalism for electron channeling in single crystals [16]. Quantitative lattice location is provided by fitting the experimental patterns with theoretical ones using a two-dimensional fit procedure [18]. Corrections for backscattered electrons that reach the detector were implemented by subtracting an isotropic background from every pattern. This backscattered electron contribution is estimated based on Geant4 simulations of electron scattering [19,20], taking into account the elemental composition and geometry of the sample, sample holder, and vacuum chamber. By probing the site occupancy of the radioactive probes as a function of annealing temperature we can investigate their thermal stability.

To this purpose a series of emission channeling experiments were performed on (Ga,Mn)As films, grown using low-temperature molecular-beam epitaxy, as described in Refs. [8,21]. The samples and experimental details are given in Table I. The quoted Mn concentrations (Mn) are estimated values based on a series of calibration measurements [23], and correspond to a density of $2.2 x \times 10^{20} \mathrm{~cm}^{-3}$, where $x$ is the quoted $(\mathrm{Mn})$. The results from samples $\mathrm{A}$ and $\mathrm{B}$ can be directly compared regarding the thermal stability of ${ }^{56} \mathrm{Mn}$ in $(\mathrm{Ga}, \mathrm{Mn}) \mathrm{As}$ with two different concentrations. The ${ }^{73}$ As experiment, on sample $\mathrm{C}$, was performed to probe the stability of the GaAs host lattice when annealing up to high temperatures $\left(\sim 600^{\circ} \mathrm{C}\right)$. All implantations were performed at room temperature and subsequent measurements were carried out in the as-implanted state and after thermal annealing in steps of $50{ }^{\circ} \mathrm{C}\left(100^{\circ} \mathrm{C}\right.$ for the ${ }^{73} \mathrm{As}$ experiment $)$ starting at $100^{\circ} \mathrm{C}$. Each annealing step was performed in high vacuum $\left(<10^{-5} \mathrm{mbar}\right)$ for $10 \mathrm{~min}$. Angular-dependent emission patterns were recorded along four crystallographic axes $(\langle 100\rangle$, $\langle 111\rangle,\langle 110\rangle$, and $\langle 211\rangle)$ at room temperature.

\section{RESULTS AND DISCUSSION}

Emission channeling results are presented and discussed in two parts starting with the identification of which lattice sites Mn occupies in (Ga,Mn)As (Sec. III A), followed by the analysis of Mn diffusion and thermal stability for both Mn concentrations (Sec. III B). This analysis includes a model for the diffusion of both $\mathrm{Mn}_{\mathrm{s}}$ and $\mathrm{Mn}_{\mathrm{i}}$, as well as a discussion of its implications on the understanding of Mn thermal stability in (Ga,Mn)As.

\section{A. Identification of the occupied lattice sites}

In (Ga,Mn)As the Mn dopants occupy mainly Gasubstitutional sites $S_{\mathrm{Ga}}$. Fitting calculated $S_{\mathrm{Ga}}$ patterns to our experimental results gives by far the best agreement. By including in the fitting tetrahedral interstitial sites with As closest neighbors $\left(T_{\mathrm{As}}\right)$, the best fit was observed for the $S_{\mathrm{Ga}}+T_{\mathrm{As}}$ double occupancy patterns, with larger fractions of ${ }^{56} \mathrm{Mn}$ probes occupying the $S_{\mathrm{Ga}}$ sites and smaller fractions the $T_{\mathrm{As}}$ sites. This is consistent with previous emission channeling experiments showing that interstitial Mn occupies only the $T_{\mathrm{As}}$ site, with $<0.5 \%$ of Mn occupying $T_{\mathrm{Ga}}$ sites [24].

As an example of the good match between experiment and simulated patterns, Fig. 1 compares the normalized experimental $\beta^{-}$emission yields for the ${ }^{56} \mathrm{Mn}$ experiment on sample B, after an annealing step at a temperature of $350{ }^{\circ} \mathrm{C}$, along the four measured directions [(a)-(d)] with the best fits to theoretical patterns [(e)-(h)]. Site fractions for the best fit, averaged over $\langle 110\rangle$ and $\langle 211\rangle$ directions, give $65 \%$ of the ${ }^{56} \mathrm{Mn}$ atoms in $S_{\mathrm{Ga}}$ sites and $14 \%$ in $T_{\mathrm{As}}$ sites. An equivalent figure for sample $\mathrm{A}$ is provided in the Supplemental Material (Fig. S1) [25]. For the radioactive ${ }^{73}$ As experiment in sample $\mathrm{C}$, the probes were found to occupy exclusively substitutional $S_{\text {As }}$ sites, with a site fraction of $\approx 100 \%$. Further details are given in the Supplemental Material [25]. For all experiments presented in Table I, fractions of other possible interstitial sites are estimated to be below $5 \%$, i.e., below the measurement uncertainty.

\section{B. Thermal stability of interstitial and substitutional Mn}

Associated with each site is its occupancy, i.e., the fraction incorporated in the respective site with respect to all the implanted ${ }^{56} \mathrm{Mn}$ (or ${ }^{73} \mathrm{As}$ ). Site fractions associated with the stable isotopes incorporated during growth will most likely 

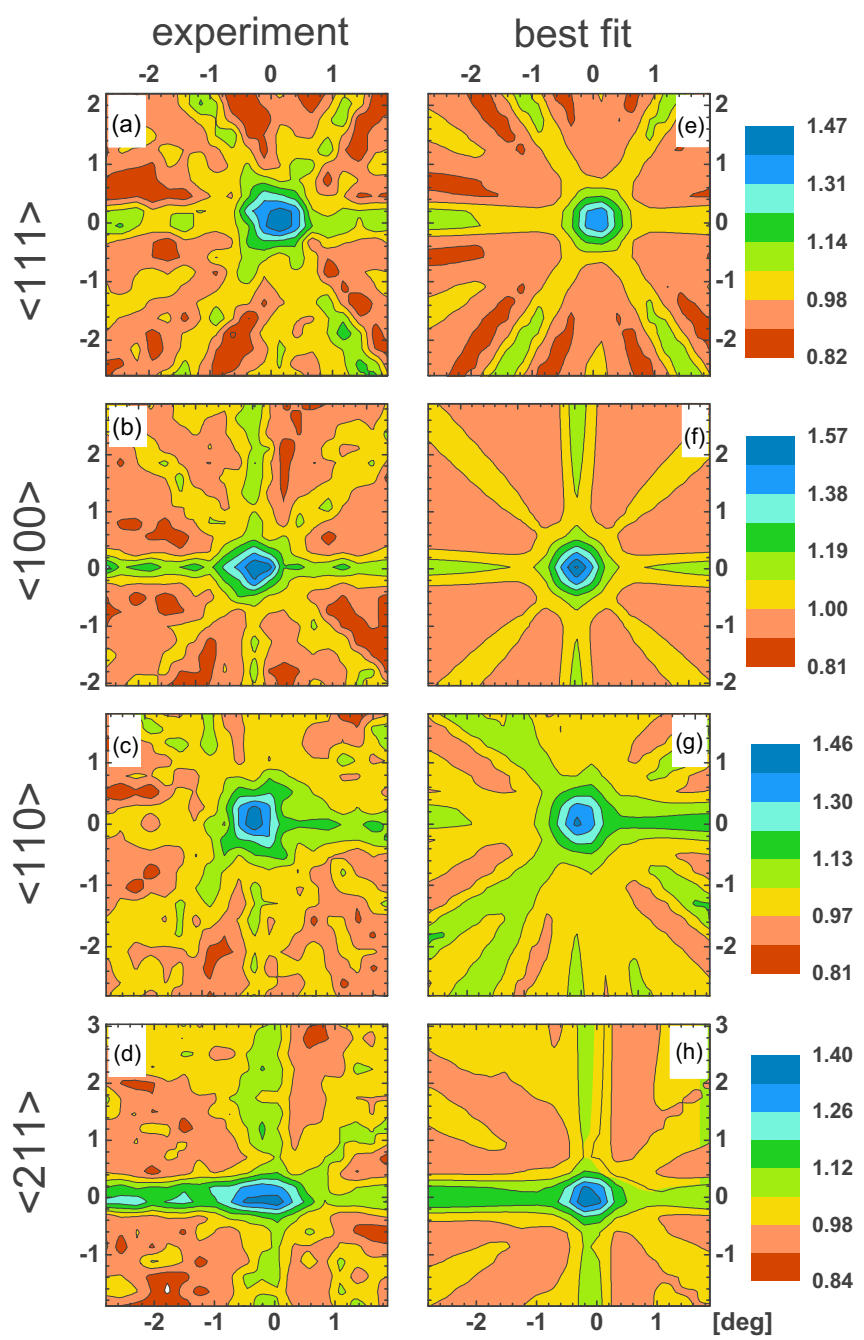

FIG. 1. (a)-(d) Normalized experimental ${ }^{56} \mathrm{Mn}$ emission channeling patterns in the vicinity of the $\langle 111\rangle,\langle 100\rangle,\langle 110\rangle$, and $\langle 211\rangle$ directions of the (Ga,Mn)As film with $5 \% \mathrm{Mn}$, following annealing at $350{ }^{\circ} \mathrm{C}$. (e)-(h) Corresponding best fits. Fit results averaged over $\langle 110\rangle$ and $\langle 211\rangle$ directions yield $65 \%$ and $14 \%$ of the $\mathrm{Mn}$ atoms on $S_{\mathrm{Ga}}$ and $T_{\mathrm{As}}$ sites, respectively.

differ, since the kinetics of implantation is very different from that of doping during LT-MBE growth. Once the Mn atoms are at rest their behavior does not depend on their history or isotope, and although the absolute site fractions may differ, the diffusion behavior we investigate here is accurately reproduced by the ${ }^{56} \mathrm{Mn}$ probes. Performing this experiment after several annealing steps provides information on the thermal stability of the occupied lattice sites.

Figure 2(a) shows the results from emission channeling experiments performed on samples $\mathrm{A}-\mathrm{C}$, as a function of annealing temperature in comparison with the ultradilute case investigated in Ref. [26] [Fig. 2(b)]. For each experiment the fitted fractions of ${ }^{56} \mathrm{Mn}$ or ${ }^{73} \mathrm{As}$ probes are plotted for the corresponding lattice sites they occupy, $S_{\mathrm{Ga}}$ and $T_{\mathrm{As}}$ for ${ }^{56} \mathrm{Mn}$, and $S_{\mathrm{As}}$ for ${ }^{73}$ As.

In emission channeling experiments, and in particular for (Ga,Mn)As thin films, two phenomena may induce changes in the obtained site fractions: (i) the annealing of implantation
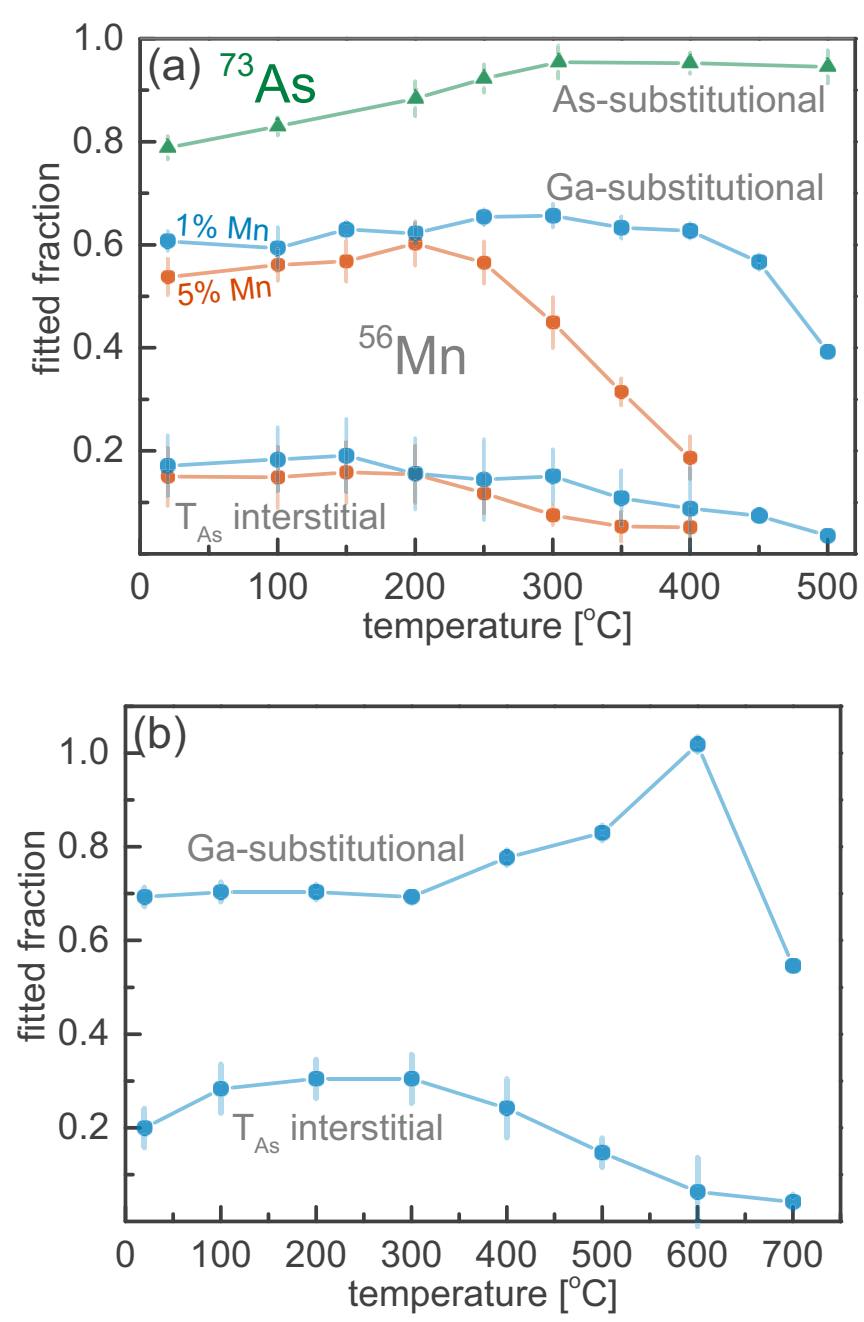

FIG. 2. (a) Fitted fractions of implanted ${ }^{73}$ As (green triangles) and ${ }^{56} \mathrm{Mn}$ (blue and orange circles) probes on (Ga,Mn)As samples doped with different concentrations of Mn, averaged over the $\langle 110\rangle$ and $\langle 211\rangle$ directions. (b) Fitted fractions of implanted ${ }^{56} \mathrm{Mn}$ probes on semi-insulating GaAs, extracted from Ref. [26].

induced damage, and (ii) diffusion processes of the implanted probes. The first scenario (i) is observed in (Ga,Mn)As at relatively low annealing temperatures, and while the thermal energy is not sufficient to induce diffusion of $\mathrm{Mn}$ atoms, it induces crystalline recovery. In other words, during implantation many defects are created, such as Ga and As vacancies $\left(V_{\mathrm{Ga}}\right.$ and $\left.V_{\mathrm{As}}\right)$ and disordered regions, where radioactive ${ }^{56} \mathrm{Mn}$ probes may become trapped. Probes trapped in these regions emit electrons which will not be channeled due to the irregular potential surrounding them and will reach the detector with an isotropic distribution. On the other hand, radioactive probes can also combine with vacancies $\left(V_{\mathrm{Ga}}\right.$ or $\left.V_{\mathrm{As}}\right)$, contributing to an increase in substitutional site fractions. Annealing of implantation damage is visible in Fig. 2(a), where substitutional fractions increase with increasing annealing temperatures up to $300{ }^{\circ} \mathrm{C}$, particularly noticeable in the ${ }^{73} \mathrm{As}$ experiment where the $S_{\mathrm{As}}$ fitted fraction increases to near $100 \%$. The second phenomenon (ii), the diffusion of implanted probes, may influence site fractions via two separate mechanisms. On the one hand, once radioactive probes become mobile and 
diffuse away from their initial position in the sample, this may lead to their incorporation in a different type of lattice site. This has been observed for instance for ${ }^{56} \mathrm{Mn}$ implanted into pure GaAs [26,27] [cf. Fig. 2(b)], where the onset of the migration of interstitial ${ }^{56} \mathrm{Mn}_{\mathrm{i}}$ leads to its incorporation in substitutional Ga sites as ${ }^{56} \mathrm{Mn}_{\mathrm{s}}$ via the combination with $\mathrm{Ga}$ vacancies. On the other hand, diffusion also leads to changes in the depth profile of the implanted probes. In that respect, although diffusion occurs in every direction within the film, due to the nature of emission channeling only diffusion towards the surface or deeper into the sample will have an impact on the measured fractions. Electrons emitted from deeper within the sample (diffusion to the bulk) are subjected to stronger dechanneling effects, whereas electrons emitted from the first few atomic layers (diffusion towards the surface) do not experience channeling effects. In both cases electrons reach the detector as an isotropic contribution resulting in a decrease in the fitted fractions. We discuss this effect in more detail below, in the context of a Mn diffusion model. A potential third scenario would be that accumulation of interstitial Mn at the surface, and resulting formation of a disordered surface layer, would enhance electron dechanneling and therefore result in an overall decrease in fitted fractions. We can however conclude that, even if this process occurs, it cannot account for the observed decrease in fitted substitutional fraction. First, Fig. S3 (in the Supplemental Material [25]) shows a recovery (an increase) in substitutional fractions upon reimplantation of ${ }^{56} \mathrm{Mn}$; if a dechanneling-enhancing layer had been formed upon high-temperature annealing, its effect (a decreased fitted fraction) would still be present after reimplantation, but the opposite is observed. Second, in our previous experiments [24] upon annealing at $200^{\circ} \mathrm{C}$ in air for $3 \mathrm{~h}$, which is known to be more effective towards the formation of a Mn-rich surface layer compared to annealing in vacuum (as in the present case), no decrease in substitutional fraction was observed despite the significant decrease in interstitial fraction. We can therefore safely exclude that the observed decrease in substitutional fraction with increasing annealing temperature is due to enhanced dechanneling caused by Mn accumulation at the surface.

Comparing the ${ }^{56} \mathrm{Mn}$ site fractions to our previous studies in pure GaAs, in Fig. 2(b), one can observe that no pronounced ${ }^{56} \mathrm{Mn}$ site changes from interstitial to substitutional sites are observed in $(\mathrm{Ga}, \mathrm{Mn}) \mathrm{As}$. In GaAs, a decrease in interstitial ${ }^{56} \mathrm{Mn}$ fraction was accompanied by a corresponding increase in substitutional fraction. We attribute the absence of this effect to the fact that the $(\mathrm{Ga}, \mathrm{Mn})$ As samples are saturated with stable $\mathrm{Mn}_{\mathrm{i}}$, incorporated during growth, which fill essentially all Ga vacancies created during implantation. Since the concentration of stable $\mathrm{Mn}_{\mathrm{i}}$ is orders of magnitude larger than the implanted ${ }^{56} \mathrm{Mn}$, only a negligible fraction of radioactive ${ }^{56} \mathrm{Mn}$ probes participate in this process, and therefore the site change is not observed for ${ }^{56} \mathrm{Mn}$. In addition, while in GaAs ${ }^{56} \mathrm{Mn}$ on substitutional Ga sites was completely stable against annealing up to a temperature of $600^{\circ} \mathrm{C}$, in $(\mathrm{Ga}, \mathrm{Mn}) \mathrm{As}$ the substitutional fraction starts to decrease at much lower temperatures. Moreover, there is a clear distinction between the experiments performed in samples A (1\% Mn) and B (5\% $\mathrm{Mn}$ ), showing that the thermal stability of both substitutional and interstitial sites decreases with Mn concentration.
TABLE II. Estimated activation energies $E_{a}$ for substitutional and interstitial Mn diffusion in (Ga,Mn)As with different Mn concentrations. $T_{1 / 2}$ represents the temperature range at which our fitted fractions reduce to $f_{0} / 2 . T_{1 / 2}$ is described in more detail in the Supplemental Material [25].

\begin{tabular}{lccccc}
\hline \hline & \multicolumn{2}{c}{ Substitutional } & & \multicolumn{2}{c}{ Interstitial } \\
\cline { 2 - 3 } \cline { 5 - 6 }$\% \mathrm{Mn}$ & $T_{1 / 2}\left({ }^{\circ} \mathrm{C}\right)$ & $E_{a}^{s}(\mathrm{eV})$ & & $T_{1 / 2}\left({ }^{\circ} \mathrm{C}\right)$ & $E_{a}^{i}(\mathrm{eV})$ \\
\hline 0.05 & 700 & 2.9 & & $450-550$ & $1.7-2.3$ \\
1 & $500-550$ & 2.4 & & $350-450$ & $1.6-2.0$ \\
5 & $350-400$ & 1.9 & & $250-300$ & $1.3-1.6$ \\
\hline \hline
\end{tabular}

Ultimately, the difference in temperature at which the $\mathrm{Mn}$ on substitutional sites becomes mobile for both samples A and B, represented in Fig. 2, is related to a decrease in thermal stability with increasing Mn concentration. This points to a possible onset of Mn segregation at lower temperatures and as such sets an upper limit for the optimum annealing temperature at which $T_{\mathrm{C}}$ can be maximized in $(\mathrm{Ga}, \mathrm{Mn}) \mathrm{As}$ thin films.

The thermal stability of $\mathrm{Mn}$ in $(\mathrm{Ga}, \mathrm{Mn})$ As is further addressed in the following paragraphs, with an estimate of the activation energies for diffusion of both $\mathrm{Mn}_{\mathrm{s}}$ and $\mathrm{Mn}_{\mathrm{i}}$ from the emission channeling results presented above, within an adequate diffusion model.

\section{Diffusion model}

Activation energies for Mn diffusion in $(\mathrm{Ga}, \mathrm{Mn}) \mathrm{As}$ can be estimated from emission channeling data by assuming a modified Arrhenius model to describe the changes in site fractions, shown in Fig. 2. The details of this calculation are given in the Supplemental Material [25], and the model is described in the remainder of this section. The resulting activation energies for substitutional and interstitial diffusion are presented in Table II for both Mn concentrations in the (Ga,Mn)As under study. Additionally, activation energies for ${ }^{56} \mathrm{Mn}$ diffusion in GaAs from Ref. [26] are also included for comparison. The main observation from Table II is that the activation energies $E_{a}$ decrease with increasing $\mathrm{Mn}$ concentration, for both substitutional and interstitial Mn. In this section, we discuss this observation in more detail and introduce a model for diffusion of $\mathrm{Mn}$ in $(\mathrm{Ga}, \mathrm{Mn}) \mathrm{As}$.

In the ultradilute regime $(<0.05 \% \mathrm{Mn})$, where only the implanted ${ }^{56} \mathrm{Mn}$ probes contribute to the total $\mathrm{Mn}$ concentration in GaAs, $\mathrm{Mn}_{\mathrm{i}}$ can be assumed to diffuse as a free, isolated interstitial $[28,29]$. In other words, the probability for a $\mathrm{Mn}_{\mathrm{i}}$ atom to occupy a position near a $\mathrm{Mn}_{\mathrm{s}}$ atom is very small, as Mn impurities are far apart in an ultradilute system. In this scenario the activation energy for free interstitial diffusion can then be directly related to the migration energy $E_{m}$, which is the energy barrier between neighboring interstitial sites. Since $\mathrm{Mn}_{\mathrm{i}}$ was observed to only occupy $T_{\mathrm{As}}$ sites both before and after thermal annealing in ferromagnetic $(\mathrm{Ga}, \mathrm{Mn}) \mathrm{As}$ [24], $E_{m}$ can be specifically attributed to the energy barrier between neighboring $T_{\mathrm{As}}$ interstitial sites. This scenario implies that $T_{\mathrm{Ga}}$ is a higher-energy configuration compared to $T_{\mathrm{As}}$, so that the barrier associated with a $T_{\mathrm{As}} \rightarrow T_{\mathrm{Ga}}$ jump is larger than the reverse $T_{\mathrm{Ga}} \rightarrow T_{\mathrm{As}}$. This also implies that at room 


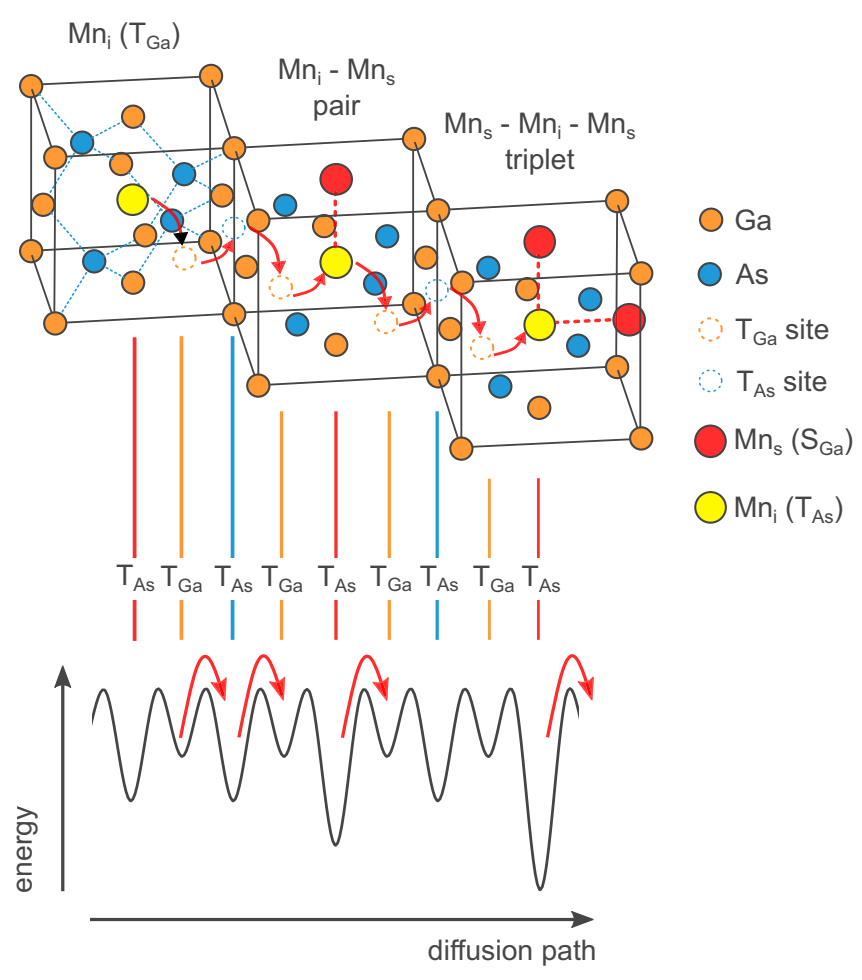

FIG. 3. Top: Interstitial $\mathrm{Mn}$ as a free interstitial, in a $\mathrm{Mn}_{\mathrm{i}}-\mathrm{Mn}_{\mathrm{s}}$ pair, and in a $\mathrm{Mn}_{\mathrm{s}}-\mathrm{Mn}_{\mathrm{i}}-\mathrm{Mn}_{\mathrm{s}}$ triplet. The red arrows indicate successive $T_{\mathrm{As}} \rightarrow T_{\mathrm{Ga}} \rightarrow T_{\mathrm{As}}$ diffusion steps between the three configurations. Bottom: Schematic representation of the energy landscape along the same diffusion path, depicting the higher stability in $T_{\mathrm{As}}$ compared to $T_{\mathrm{Ga}}$ sites, and the effect of $E_{b}$, i.e., the increase in activation energy (from $E_{m}$ for an isolated interstitial) induced by the formation of pairs $\left(\mathrm{Mn}_{\mathrm{s}}-\mathrm{Mn}_{\mathrm{i}}\right)$ and triplets $\left(\mathrm{Mn}_{\mathrm{s}}-\mathrm{Mn}_{\mathrm{i}}-\mathrm{Mn}_{\mathrm{s}}\right)$. The effect of more distant neighbors $(\Delta)$ described in the main text is not represented.

temperature the $T_{\mathrm{Ga}} \rightarrow T_{\mathrm{As}}$ barrier is overcome, resulting in the prevalence of $T_{\mathrm{As}}$ sites. Thus, we can conclude that interstitial diffusion occurs along the path $T_{\mathrm{As}} \rightarrow T_{\mathrm{Ga}} \rightarrow T_{\mathrm{As}}$, as depicted in Fig. 3 and its activation energy is directly linked with $E_{m}, E_{a}=E_{m}$.

In the higher concentration regime investigated in this paper, the interstitial diffusion cannot be solely modeled by $E_{a}=$ $E_{m}$, as a considerable fraction of the interstitials are trapped by neighboring substitutional $\mathrm{Mn}$, forming pairs $\left(\mathrm{Mn}_{\mathrm{s}}-\mathrm{Mn}_{\mathrm{i}}\right)$ and triplets $\left(\mathrm{Mn}_{\mathrm{s}}-\mathrm{Mn}_{\mathrm{i}}-\mathrm{Mn}_{\mathrm{s}}\right)$ (cf. Fig. 3). These complexes contribute to the activation energy for interstitial diffusion with $E_{b}$, an additional energy barrier that $\mathrm{Mn}_{\mathrm{i}}$ has to overcome to break the bond with substitutional Mn, resulting in $E_{a}=E_{m}+$ $E_{b}$. Following a purely statistical approach, as described in Ref. [30], i.e., assuming that the substitutional Mn is randomly distributed in the lattice, the fractions of isolated substitutional Mn atoms $\left(x_{\mathrm{s}}\right)$ and of pairs of neighboring substitutional Mn atoms $\left(x_{\mathrm{s}-\mathrm{s}}\right)$ as a function of Mn concentration $x$ can be calculated by $x_{\mathrm{s}}=(1-x)^{12}$ and $x_{\mathrm{s}-\mathrm{s}}=12 x(1-x)^{18}$. For $1 \%$ $\mathrm{Mn}$, this gives $x_{\mathrm{s}}=0.89$ and $x_{\mathrm{s}-\mathrm{s}}=0.10$. For $5 \%, x_{\mathrm{s}}=0.54$ and $x_{\mathrm{s}-\mathrm{s}}=0.24$.

In other words, the probability of interstitial $\mathrm{Mn}_{\mathrm{i}}$ to be trapped by complexes involving more than one substitutional $\mathrm{Mn}_{\mathrm{s}}$ is considerably enhanced for $(\mathrm{Mn})=5 \% . E_{b}^{i}$ can then be described as an energy barrier that is largely determined by the Coulomb interaction between oppositely charged $\mathrm{Mn}_{\mathrm{s}}$ and $\mathrm{Mn}_{\mathrm{i}}$ defects, and should, intuitively, increase with the number of $\mathrm{Mn}_{\mathrm{s}}$ atoms involved in the complex. Density functional theory (DFT) calculations have indeed predicted this effect, with binding energies of $E_{b}^{\text {pair }}=0.49 \mathrm{eV}$ for the $\operatorname{Mn}_{\mathrm{i}}\left(T_{\mathrm{As}}\right)-\mathrm{Mn}_{\mathrm{s}}$ bond, and $E_{b}^{\text {triplet }}=0.81 \mathrm{eV}$ for $\mathrm{Mn}_{\mathrm{s}}-\mathrm{Mn}_{\mathrm{i}}\left(T_{\mathrm{As}}\right)-\mathrm{Mn}_{\mathrm{s}}$ bonds [13]. The complete diffusion model, up to this point, can then be illustrated as in Fig. 3.

Given the statistical prevalence of Mn complexes at higher concentrations, one would expect the activation energy for interstitial diffusion to increase with Mn content. However, the emission channeling results presented here show the contrary, i.e., the activation energy for diffusion of interstitial Mn decreases with Mn concentration (cf. Table II), which clearly points towards an additional diffusion enhancing mechanism. We propose that, while increasing Mn concentration adds a binding contribution to the activation energy for interstitial diffusion, associated with the Coulomb interaction between a single $\mathrm{Mn}_{\mathrm{s}}$ and a $\mathrm{Mn}_{\mathrm{i}}$ atom, the same Coulomb interaction between the interstitial and more distant $\mathrm{Mn}_{\mathrm{s}}$ defects decreases the activation energy by an amount $\Delta$, thus enhancing diffusion. The Coulomb interaction energy between $\mathrm{Mn}_{\mathrm{s}}^{-}$and $\mathrm{Mn}_{\mathrm{i}}^{2+}$ at a distance of $10 \AA$ can be estimated as $\Delta \approx 0.2 \mathrm{eV}$, taking the GaAs dielectric constant (13.2). However, within a distance of $10 \AA$ in a $5 \%$ Mn sample there are six $\mathrm{Mn}_{\mathrm{s}}$ in the neighborhood of each $\mathrm{Mn}_{\mathrm{i}}$, resulting in possible potential modifications of $\Delta \approx 1.2 \mathrm{eV}$. A similar estimation can be performed for a $1 \%$ sample, resulting in an interaction energy of $\Delta \approx 0.8 \mathrm{eV}$. These energies can effectively compete with $E_{b}^{i}$, ultimately decreasing the activation energy for interstitial diffusion, $E_{a}=E_{m}+E_{b}-\Delta$.

Comparing values for $E_{a}^{i}$ in Table II with $E_{b}^{i}$ from Ref. [13], discussed above, suggests that the activation energy for interstitial diffusion $E_{a}^{i}$ decreases from the ultradilute regime to $5 \% \mathrm{Mn}$ by $\Delta E_{a}^{i} \sim 0.1-1.0 \mathrm{eV}$, driven essentially by the competing contributions from $E_{b}^{i}$ and $\Delta$, assuming a similar $E_{m}$. However, $E_{m}$ requires additional discussion. In the ultradilute regime $E_{a}^{i}$ is entirely due to $E_{m}\left(E_{a}^{i}=E_{m}\right)$, and $E_{a}^{i}$ is in fact larger than the values determined here for $1 \%$ and $5 \%$. Therefore, $E_{m}$ may also decrease from the ultradilute to the few percent regime. A possible contribution to this decrease is the lattice expansion induced by the Mn doping, i.e., that interstitial Mn experiences a lower migration barrier in a more open lattice, although the increase in lattice constant from the ultradilute case to $5 \% \mathrm{Mn}$ in (Ga,Mn)As layers is only $0.2 \%$ [23]). Nevertheless, even if this decrease affects $E_{m}$, it alone cannot explain all the observed changes in $E_{a}^{i}$; in that case, $E_{a}^{i}$ would vary by the same amount, which is not observed. In order to explain the additional variation, $\Delta$ is required.

The diffusion of substitutional Mn, on the other hand, can be assumed to be of the Frank-Turnbull type, i.e., thermally activated $\mathrm{Mn}_{\mathrm{s}}$ first leaves its substitutional lattice site and then diffuses as an interstitial until it encounters the next stable trap, usually a Ga vacancy. At sufficiently high $\mathrm{Mn}$ concentrations, also regions with segregated $\mathrm{Mn}$ can act as traps. The activation energy for this type of diffusion can be split in two terms, one consisting of the binding energy 
$E_{b}\left(\mathrm{Mn}_{\mathrm{s}}\right)$ of $\mathrm{Mn}$ to the substitutional site, the other being the activation energy $E_{a}^{i}$ for interstitial diffusion of $\mathrm{Mn}_{\mathrm{i}}$, resulting in a total activation energy of $E_{a}^{s}=E_{a}^{i}+E_{b}\left(\mathrm{Mn}_{\mathrm{s}}\right)$. Note that for this type of diffusion mechanism, an increase or a decrease of the activation energy for interstitial diffusion will also lead to a corresponding effect on the stability of the substitutional Mn species. As established above, $\Delta$ drives down the activation energy for interstitial diffusion and, consequently, also for substitutional diffusion, in accordance with the results in Table II.

A different model for the diffusion of $\mathrm{Mn}$ in $(\mathrm{Ga}, \mathrm{Mn}) \mathrm{As}$ has been proposed in Refs. [31,32], which considers that clustering of Mn occurs via pure substitutional diffusion driven solely by $\mathrm{Ga}$ vacancies $\left(V_{\mathrm{Ga}}\right)$. The weakness of this model is that it neglects the interaction of $\mathrm{Mn}$ interstitials with $\mathrm{Ga}$ vacancies, i.e., the fact that interstitial $\mathrm{Mn}$, once it becomes mobile, easily converts to substitutional by filling up existing $V_{\mathrm{Ga}}$ (which is associated with an energy gain). The reverse process, i.e., dissociation of $\mathrm{Mn}_{\mathrm{s}}$ by becoming interstitial and leaving behind a $V_{\mathrm{Ga}}$, is also possible, though only with higher thermal activation energy, cf. the discussion above. In contrast, it is assumed in Refs. [31,32] that Mn interstitials have been completely driven out from the sample before clustering of substitutional $\mathrm{Mn}$ atoms starts. Moreover, the substitutional diffusion of $\mathrm{Mn}$ is assumed to be the consequence of a rather high concentration of mobile $V_{\mathrm{Ga}}\left(\approx 1 \times 10^{18} \mathrm{~cm}^{-3}\right)$, which is not explained by the model itself but given as external input. The assumption that interstitial out-diffusion of $\mathrm{Mn}$ in (Ga,Mn)As can be entirely completed before any substitutional diffusion starts is contrary to what we observe in our emission channeling experiments, especially in the 5\% Mn sample, where the decrease in interstitial and substitutional Mn fractions takes place in overlapping temperature regimes, i.e., the two processes occur simultaneously, though slower for substitutional Mn.

\section{Substitutional Mn diffusion as a limiting factor}

The temperature at which substitutional Mn becomes mobile changes from $700^{\circ} \mathrm{C}$ in the ultradilute regime, to $\sim 500^{\circ} \mathrm{C}$ at $1 \%(\mathrm{Mn})$, to $\sim 350^{\circ} \mathrm{C}$ at $5 \%(\mathrm{Mn})$. Although the formation of MnAs precipitates is well documented in the $400-600^{\circ} \mathrm{C}$ temperature range $[11,12,14,15]$, our data show that this segregation process can already take place at lower temperatures, likely with the formation of disordered Mn-rich regions preceding well-defined secondary phases. This scenario is further supported by the emission channeling data obtained upon reimplanting ${ }^{56} \mathrm{Mn}$ after high-temperature annealing $\left(400^{\circ} \mathrm{C}\right.$ for $5 \%$ and $500^{\circ} \mathrm{C}$ for $1 \% \mathrm{Mn}$ ), i.e., after diffusion of a significant fraction of substitutional Mn. A thorough analysis of this effect is beyond the scope of this paper and is therefore only briefly described in the Supplemental Material [25].

At several \% Mn, segregation can occur at annealing temperatures as low as $200^{\circ} \mathrm{C}$, counteracting the improvement of $T_{\mathrm{C}}$ by removal of interstitials, introducing an upper limit for the optimum annealing temperature. On the other hand, the lower limit is determined by the removal of interstitial $\mathrm{Mn}$. From our results (Table II), the activation energy $E_{a}$ for interstitial diffusion also decreases with Mn concentration, although less significantly than for substitutional. The annealing temperature window for $T_{\mathrm{C}}$ and magnetization optimization in $(\mathrm{Ga}, \mathrm{Mn}) \mathrm{As}$ is therefore narrowed and limited by two phenomena which have opposite effects on $T_{\mathrm{C}}$ : interstitial out-diffusion and substitutional low-temperature segregation. Although these two processes are governed by rather close activation energies, it is possible to induce the diffusion of interstitial $\mathrm{Mn}$ (with a lower activation energy) while minimizing substitutional segregation, as long as the thermal annealing is sufficiently long and at a suitably low temperature (typically several hours for films $\sim 25 \mathrm{~nm}$ at temperatures of $160-180^{\circ} \mathrm{C}$ ) [9]. In this picture, film thickness also plays an important role. Decreasing the thickness also decreases the diffusion distance required for interstitial $\mathrm{Mn}$ to be passivated at the surface. In contrast, the segregation of substitutional Mn is in principle unaffected by a reduction in thickness since the required diffusion length is determined in that case by the (local) Mn concentration. Therefore, thinner films favor passivation of interstitial $\mathrm{Mn}$ and, indeed, the highest $T_{\mathrm{C}}$ and magnetization are observed in thinner films, typically $\sim 25 \mathrm{~nm}[9]$.

\section{CONCLUSION}

This work addresses the lattice location and thermal stability of $\mathrm{Mn}$ in ferromagnetic $(\mathrm{Ga}, \mathrm{Mn})$ As doped with $1 \%$ and 5\% Mn. The majority of Mn atoms have been found to substitute Ga sites, while a significant fraction occupies tetrahedral interstitial sites with As nearest neighbors.

The thermal stability of both substitutional and interstitial Mn decrease with Mn concentration, with estimated activation energies for diffusion of interstitial $\mathrm{Mn}$ at $1.6-2.0 \mathrm{eV}$ for $1 \%$ and $1.3-1.6 \mathrm{eV}$ for $5 \% \mathrm{Mn}$, and of substitutional $\mathrm{Mn}$ at 2.4 and $1.9 \mathrm{eV}$, respectively. This decrease in thermal stability at a few $\% \mathrm{Mn}$ content is directly related to an increased Mn diffusivity in $(\mathrm{Ga}, \mathrm{Mn}) \mathrm{As}$ films, which we attribute to the Coulomb interactions between substitutional Mn and diffusing interstitial Mn atoms.

These findings shed light on our understanding of Mn selfcompensation in $(\mathrm{Ga}, \mathrm{Mn})$ As. The delicate balance between interstitial and substitutional thermal stability defines the narrow temperature window in which the Curie temperature and the magnetization can be maximized. The lower limit is defined by the mobility of Mn interstitials towards passivation at the surface, whereas the higher limit is limited by the mobility of substitutional Mn, i.e., the onset of Mn segregation into secondary phases. Since the Mn concentration affects more strongly the upper limit, the optimal annealing temperature window narrows down with increasing Mn concentration.

\section{ACKNOWLEDGMENTS}

This work was supported by the Fund for Scientific Research-Flanders, the Concerted Research Action of the KU Leuven BOF (Grants No. GOA/14/007, No. STRT/14/002, No. C12/18/006, and No. C14/18/074), the Portuguese Foundation for Science and Technology (CERN/FIS-PAR/0005/2017), and the European Union's Horizon 2020 under Grants Agreement No. 654002 (ENSAR2) and No. 824096 (RADIATE). T.A.L.L. acknowledges the Research Foundation Flanders (FWO) for his Ph.D. fellowship (File No. 52152 and No. 29681). 
[1] T. Dietl and H. Ohno, Dilute ferromagnetic semiconductors: Physics and spintronic structures, Rev. Mod. Phys. 86, 187 (2014).

[2] T. Jungwirth, J. Wunderlich, V. Novák, K. Olejník, B. L. Gallagher, R. P. Campion, K. W. Edmonds, A. W. Rushforth, A. J. Ferguson, and P. Němec, Spin-dependent phenomena and device concepts explored in (Ga,Mn)As, Rev. Mod. Phys. 86, 855 (2014).

[3] T. Dietl, A ten-year perspective on dilute magnetic semiconductors and oxides, Nat. Mater. 9, 965 (2010).

[4] M. Dobrowolska, K. Tivakornsasithorn, X. Liu, J. K. Furdyna, M. Berciu, K. M. Yu, and W. Walukiewicz, Controlling the Curie temperature in $(\mathrm{Ga}, \mathrm{Mn})$ As through location of the Fermi level within the impurity band, Nat. Mater. 11, 444 (2012).

[5] T. Jungwirth, K. Y. Wang, J. Mašek, K. W. Edmonds, J. König, J. Sinova, M. Polini, N. A. Goncharuk, A. H. MacDonald, M. Sawicki et al., Prospects for high temperature ferromagnetism in (Ga,Mn)As semiconductors, Phys. Rev. B 72, 165204 (2005).

[6] K. M. Yu, W. Walukiewicz, T. Wojtowicz, I. Kuryliszyn, X. Liu, Y. Sasaki, and J. K. Furdyna, Effect of the location of Mn sites in ferromagnetic $\mathrm{Ga}_{1-x} \mathrm{Mn}_{x}$ As on its Curie temperature, Phys. Rev. B 65, 201303(R) (2002).

[7] M. Wang, K. W. Edmonds, B. L. Gallagher, A. W. Rushforth, O. Makarovsky, A. Patanè, R. P. Campion, C. T. Foxon, V. Novak, and T. Jungwirth, High Curie temperatures at low compensation in the ferromagnetic semiconductor (Ga,Mn)As, Phys. Rev. B 87, 121301(R) (2013).

[8] P. Němec, V. Novák, N. Tesařová, E. Rozkotová, H. Reichlová, D. Butkovičová, F. Trojánek, K. Olejník, P. Malý, R. P. Campion et al., The essential role of carefully optimized synthesis for elucidating intrinsic material properties of ( $\mathrm{Ga}, \mathrm{Mn}) \mathrm{As}$, Nat. Commun. 4, 1422 (2013).

[9] M. Wang, R. P. Campion, A. W. Rushforth, K. W. Edmonds, C. T. Foxon, and B. L. Gallagher, Achieving high Curie temperature in (Ga,Mn)As, Appl. Phys. Lett. 93, 132103 (2008).

[10] K. W. Edmonds, P. Bogusławski, K. Y. Wang, R. P. Campion, S. N. Novikov, N. R. S. Farley, B. L. Gallagher, C. T. Foxon, M. Sawicki, T. Dietl et al., Mn Interstitial Diffusion in (Ga,Mn)As, Phys. Rev. Lett. 92, 037201 (2004).

[11] M. Yokoyama, H. Yamaguchi, T. Ogawa, and M. Tanaka, Zincblende-type MnAs nanoclusters embedded in GaAs, J. Appl. Phys. 97, 10D317 (2005).

[12] A. Kwiatkowski, D. Wasik, M. Kamińska, R. Bożek, J. Szczytko, A. Twardowski, J. Borysiuk, J. Sadowski, and J. Gosk, Structure and magnetism of MnAs nanocrystals embedded in GaAs as a function of post-growth annealing temperature, J. Appl. Phys. 101, 113912 (2007).

[13] V. I. Baykov, P. A. Korzhavyi, and B. Johansson, Diffusion of Interstitial $\mathrm{Mn}$ in the Dilute Magnetic Semiconductor (Ga,Mn)As: The Effect of a Charge State, Phys. Rev. Lett. 101, 177204 (2008).

[14] J. Sadowski, J. Z. Domagala, R. Mathieu, A. Kovács, T. Kasama, R. E. Dunin-Borkowski, and T. Dietl, Formation process and superparamagnetic properties of $(\mathrm{Mn}, \mathrm{Ga}) \mathrm{As}$ nanocrystals in GaAs fabricated by annealing of $(\mathrm{Ga}, \mathrm{Mn}) \mathrm{As}$ layers with low Mn content, Phys. Rev. B 84, 245306 (2011).

[15] A. Kovács, J. Sadowski, T. Kasama, M. Duchamp, and R. E. Dunin-Borkowski, Effect of post-growth annealing on secondary phase formation in low-temperature-grown Mn-doped GaAs, J. Phys. D: Appl. Phys. 46, 145309 (2013).

[16] H. Hofsäss and G. Lindner, Emission channeling and blocking, Phys. Rep. 201, 121 (1991).

[17] M. R. Silva, U. Wahl, J. G. Correia, L. M. Amorim, and L. M. C. Pereira, A versatile apparatus for on-line emission channeling experiments, Rev. Sci. Instrum. 84, 073506 (2013).

[18] U. Wahl, J.G. Correia, S. Cardoso, J.G. Marques, A. Vantomme, and G. Langouche, Electron emission channeling with positionsensitive detectors, Nucl. Instrum. Methods Phys. Res. B 136138, 744 (1998).

[19] S. Agostinelli, J. Allison, K. Amako, J. Apostolakis, H. Araujo, P. Dubois, M. Asai, D. Axen, S. Banerjee, G. Barrand et al., Geant4-a simulation toolkit, Nucl. Instrum. Methods Phys. Res. A 506, 250 (2003).

[20] J. Allison, K. Amako, J. Apostolakis, H. Araujo, P. Dubois, M. Asai, G. Barrand, R. Capra, S. Chauvie, R. Chytracek et al., Geant 4 developments and applications, IEEE Trans. Nucl. Sci. 53, 270 (2006).

[21] R. P. Campion, K. W. Edmonds, L. X. Zhao, K. Y. Wang, C. T. Foxon, B. L. Gallagher, and C. R. Staddon, The growth of GaMnAs films by molecular beam epitaxy using arsenic dimers, J. Cryst. Growth 251, 311 (2003).

[22] J. F. Ziegler, M. D. Ziegler, and J. P. Biersack, SRIM - The stopping and range of ions in matter (2010), Nucl. Instrum. Methods Phys. Res. Sect. B 268, 1818 (2010).

[23] L. X. Zhao, C. R. Staddon, K. Y. Wang, K. W. Edmonds, R. P. Campion, B. L. Gallagher, and C. T. Foxon, Intrinsic and extrinsic contributions to the lattice parameter of GaMnAs, Appl. Phys. Lett. 86, 071902 (2005).

[24] T. A. L. Lima, U. Wahl, V. Augustyns, D. J. Silva, A. Costa, K. Houben, K. W. Edmonds, B. L. Gallagher, R. P. Campion, M. J. Van Bael et al., Identification of the interstitial Mn site in ferromagnetic (Ga,Mn)As, Appl. Phys. Lett. 106, 012406 (2015).

[25] See Supplemental Material at http://link.aps.org/supplemental/ 10.1103/PhysRevB.100.144409 for complementary emission channeling data, and additional considerations included in the diffusion model described in the main text.

[26] L. M. C. Pereira, U. Wahl, S. Decoster, J. G. Correia, L. M. Amorim, M. R. da Silva, J. P. Araújo, and A. Vantomme, Stability and diffusion of interstitial and substitutional $\mathrm{Mn}$ in GaAs of different doping types, Phys. Rev. B 86, 125206 (2012).

[27] L. M. C. Pereira, U. Wahl, S. Decoster, J. G. Correia, M. R. da Silva, A. Vantomme, and J. P. Araújo, Direct identification of interstitial $\mathrm{Mn}$ in heavily p-type doped GaAs and evidence of its high thermal stability, Appl. Phys. Lett. 98, 201905 (2011).

[28] L. M. C. Pereira, J. P. Araújo, M. J. Van Bael, K. Temst, and A. Vantomme, Practical limits for detection of ferromagnetism using highly sensitive magnetometry techniques, J. Phys. D: Appl. Phys. 44, 215001 (2011). 
[29] L. M. C. Pereira, U. Wahl, J. G. Correia, S. Decoster, L. M. Amorim, M. R. da Silva, J. P. Araújo, and A. Vantomme, Evidence of $\mathrm{N}$ substitution by Mn in GaN, Phys. Rev. B 86, 195202 (2012).

[30] R. E. Behringer, Number of single, double, and triple clusters in a system containing two types of atoms, J. Chem. Phys. 29, 537 (1958).
[31] H. Raebiger, M. Ganchenkova, and J. von Boehm, Diffusion and clustering of substitutional $\mathrm{Mn}$ in (Ga,Mn)As, Appl. Phys. Lett. 89, 012505 (2006).

[32] T. Hynninen, M. Ganchenkova, H. Raebiger, and J. von Boehm, Ferromagnetism and its evolution during longterm annealing in (Ga,Mn)As, Phys. Rev. B 74, 195337 (2006). 